Jonas de Vylder; Dominique Van Der Straeten; Wilfried Philips, "Multiple leaf tracking using computer vision methods with shape constraints",Sensing Technologies for Biomaterial, Food, and Agriculture 2013, Naoshi Kondo, Editors, 88810 , SPIE Proceedings Vol. 8881

Copyright 2013 Society of Photo Optical Instrumentation Engineers. One print or electronic copy may be made for personal use only. Systematic reproduction and distribution, duplication of any material in this paper for a fee or for commercial purposes, or modification of the content of the paper are prohibited.

http://dx.doi.org/10.1117/12.2029789 


\title{
Multiple Leaf Tracking Using Computer Vision Methods with Shape Constraints
}

\author{
Jonas De Vylder ${ }^{a}$ \\ Dominique Van Der Straeten ${ }^{b}$ \\ Wilfried Philips ${ }^{a}$ \\ ${ }^{a}$ Ghent University, TELIN - iMinds - IPI, St-Pietersnieuwstraat 41, B-9000 Ghent, Belgium; \\ ${ }^{b}$ Ghent University, Department of Physiology, Laboratory of Functional Plant Biology, \\ Ledeganckstraat 35, 9000 Gent, Belgium
}

\begin{abstract}
Accurate monitoring of leaves and plants is a necessity for research on plant physiology. To aid this biological research, we propose a new active contour method to track individual leaves in chlorophyll fluorescence time laps sequences. The proposed active contour algorithm is developed such that it can handle sequences with low temporal resolution. This paper proposes a novel optimization method which incorporates prior knowledge about the plant shape. Tests show that the proposed framework outperforms state of the art tracking methods.
\end{abstract}

Keywords: segmentation, tracking, active contours, biological monitoring

\section{INTRODUCTION}

With a worldwide growing demand for food, the pressure on farmers to produce bigger harvests is increasing. In order to get a bigger harvest, without using more land, staff and other resources, it is necessary to carefully organize the cropping. This means that the right crops and the right type of plant have to be seeded, the best fertilizer and most efficient pesticide have to be chosen, etc. This however is not a trivial task since this all depends where you want to harvest, e.g. in what type of soil or what type of climate the seeds are grown. In order to model all these factors, biological laboratories have started growing plants in controlled environments, where they can measure the influence of stress factors such as wind, drought, light conditions, etc. Under these controlled conditions, they can compare the influences of stress factors on plant growth of different types or completely different species. However measuring the well being of a plant is a challenging task. In Caerle et al. ${ }^{1}$ they have proven that monitoring plants with a chlorophyll fluorescence camera results in biological relevant information, since the measured fluorescence of plants is correlated with the photosynthesis the plant undergoes. Measuring the intensity of a plant in a chlorophyll fluorescence image is a time consuming and tedious task. It requires a skilled technician to delineate each leaf in an image. This becomes unfeasible if this has to be done for a large data set, or if biologists want to monitor plant changes over time, e.g. in a time lapse sequence. In Fig. 1 four consecutive time frames are shown of such a time lapse sequence. Note that the contrast with the background is rather good, but that delineating individual leaves is rather difficult at places where the leaves partially occlude each other. A second observation which can be seen in the last two time frames concerns the mobility of the leaves. Leaves do not only grow between two time frames, but are also able to move. This leaf motion is intrinsic to the plants, so it is not possible to avoid this motion by monitoring the plants in a wind free environment.

In this paper we propose to automate the fluorescence measurements using computer vision algorithms. An interesting framework to do this is the active contour framework, which enables to track each individual leaf. In the active contour framework, an initial contour is moved and deformed in order to minimize a specific energy function. This energy function should be minimal when the contour is delineating the object of interest, e.g. a leaf. Two main groups can be distinguished in the active contour framework: one group representing the active contour explicitly as a parametrized curve and a second group which represents the contour implicitly using e.g. level sets. In the first group, also called snakes, the contour generally converges towards edges in the image. ${ }^{2-4}$ The second group generally has an energy function based on region properties, such as the intensity variance of the enclosed segment. ${ }^{5,6}$ These geometric active contour approaches have 

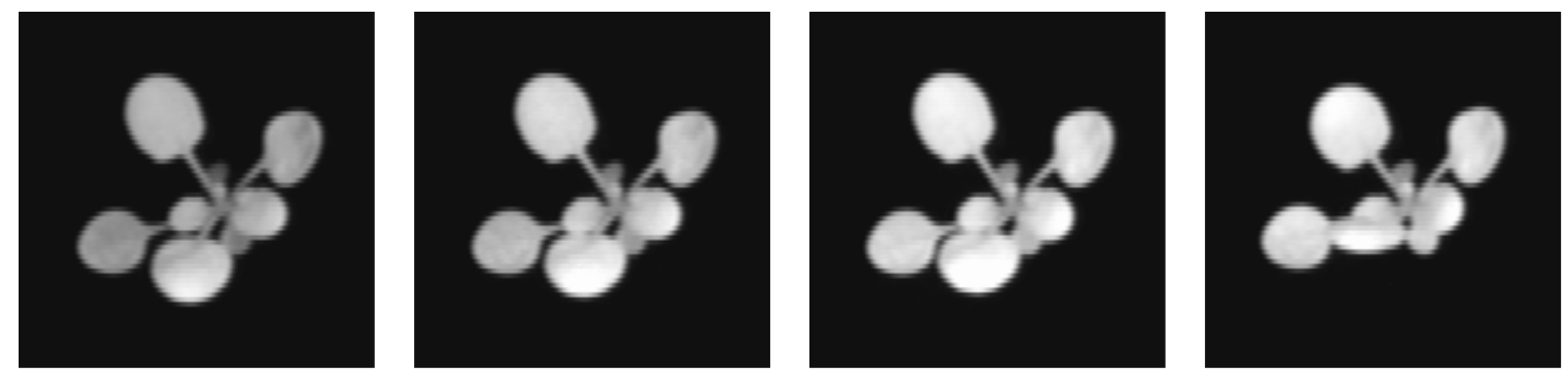

Figure 1. An example of four consecutive time frames of a time lapse sequence. This sequence monitors an Arabidopsis plant with a chlorophyll fluorescence camera.

gained a lot of interest since they have some benefits over snakes. They can for example easily change their shape, e.g. splitting a segment into multiple unconnected segments. Recently an active contour model has been proposed with a convex energy function, making it possible to define fast global optimizers. ${ }^{7,8}$

Unfortunately do the level set approaches assume certain prior knowledge about the regions defined, e.g. the variance of intensity in the segments should be minimal. These kind of assumptions are not always valid and might bias the individual leaf measurements. Therefore we will base our new active contour model on the snake approach. This doesn't form a problem since leaves don't divide and therefore we don't need the variable shape of geometric active contours. Since the snake model does not have a convex energy, extra care has to be taken in order to avoid optimization to false local minima. In this paper we propose a specific optimization scheme developed for the segmentation and tracking of individual leaves in time lapse sequences.

This paper is arranged as follows. The next section will give an overview of the different steps used by the proposed method. Section 3 explains the rosette segmentation, whereas section 4 elaborates on the leaf segmentation steps. The proposed method is tested and compared to state of the art methods in section 5. Section 6 recapitulates and concludes.

\section{OVERVIEW}

In Fig. 2 a flowchart of the proposed tracking technique is shown. The proposed method consists of three big parts: rosette segmentation, leaf detection and finally the individual leaf segmentation. The complete rosette, i.e. the full plant, is segmented out of each frame. This rosette segment serves as an aid for both the leaf detection as for the actual individual leaf segmentation step. The leaf detection step is initialized by the leaf segments of the previous frame, which are transformed such that they can serve as an initialization for the actual leaf segmentation. This transformation is based both on the current frame and on the result of the rosette segmentation.

The actual leaf segmentation is based on the active contour framework. The active contour optimization is extended such that it takes into account the segmentation result of the complete rosette. Based on the resulting segments, the average fluorescence, i.e. the amount of photosynthesis, for each leaf can be measured and analyzed. The fluorescence analysis is just mentioned for the sake of completeness, but this paper will not elaborate further on this step, since we will focus on the computer vision part.

The proposed algorithm starts once the full time lapse sequence is captured. The last frame is segmented using a watershed algorithm. This segmentation might contain errors and should be checked and if necessary corrected by a human operator. The result of the last frame will serve as an initialization for the previous frame. By starting at the end and working to the first frame, the detection of new leaves can be omitted.

\section{ROSETTE SEGMENTATION}

Since there is a clear difference between the intensity of the rosette and the background intensity, the complete rosette can be segmented using Otsu's thresholding. ${ }^{9}$ This method calculates a threshold in order to minimize the variance of the foreground, $\sigma_{f}$, and the variance of the background, $\sigma_{b}$, i.e.

$$
t=\underset{t}{\arg \min }\left(p_{f}(t) \sigma_{f}+p_{b}(t) \sigma_{b}\right)
$$




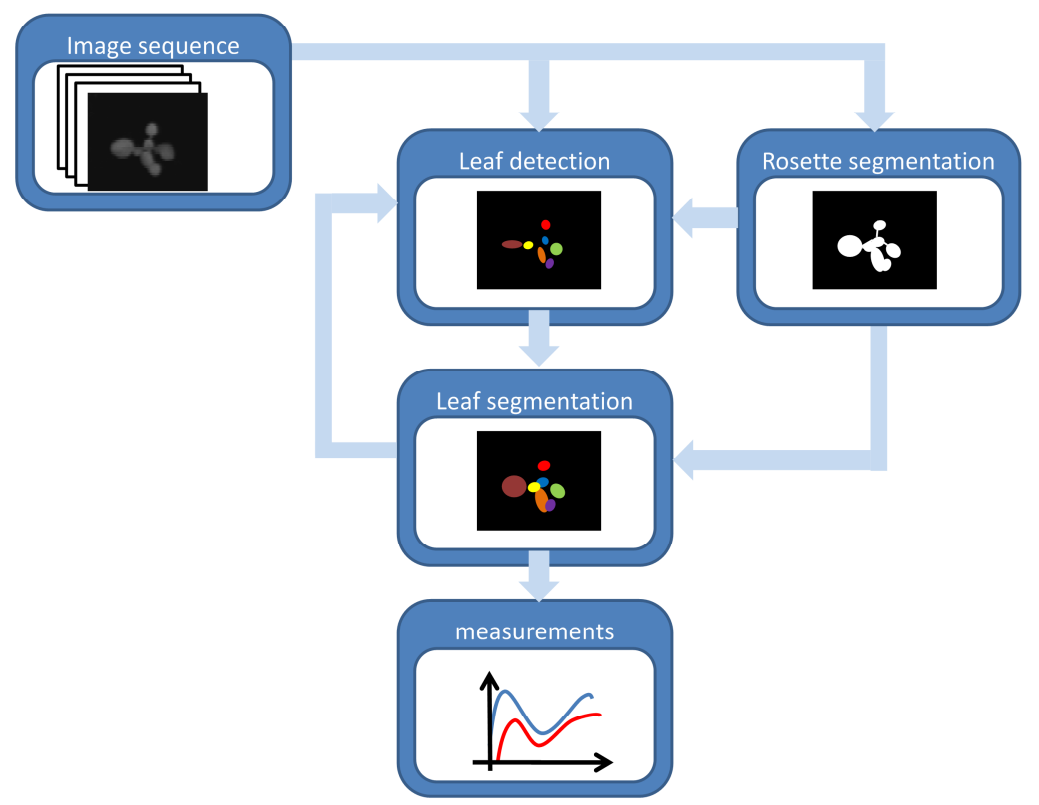

Figure 2. The main steps of the processing method.

where $p_{f}(t)$ is the probability that a pixel in the image is higher than $t$ and $p_{b}(t)$ calculates the probability the pixel is lower than $t$. For the remaining of the proposed method, it is important that all pixels belonging to the rosette are detected, whereas background pixels which are considered to be foreground have a lower influence. Therefore we propose to use a morphological closing on the threshold result. This closing will remove small errors coming from shadows or veins visible in the image. For the dataset used in this paper, a disk shaped structuring element of dimension $3 \times 3$ was used for the morphological closing. Datasets with a different resolution might need a structuring element of different size.

\section{LEAF DETECTION AND SEGMENTATION}

The segmentation of the individual leaves will happen in two steps, as been described in section 2. Both the leaf detection as the actual segmentation is based on the active contour framework. Therefore this section will first resume the basics of active contour segmentation. Then both the detection and segmentation step will be described in the remaining two subsections.

\subsection{Parametric Active Contours}

The original parametric active contour model proposed by Kass et al., ${ }^{2}$ defines the active contour as a parametric curve, $\mathbf{r}(s)=(x(s), y(s))$ for $s \in[0,1]$, which moves in the spatial domain until the energy functional in Eq. (2) reaches its minimum value.

$$
E_{\text {snake }}[\mathbf{r}(.)]=E_{\text {int }}[\mathbf{r}(.)]+E_{\text {ext }}[\mathbf{r}(.)]
$$

$E_{\text {int }}[\mathbf{r}()$.$] and E_{\text {ext }}[\mathbf{r}()$.$] represent respectively the internal and external energy of the contour. The internal energy is a$ regularization and enforces smoothness along the contour. A common internal energy function is defined as follows:

$$
E_{\text {int }}[\mathbf{r}(.)]=\frac{1}{2} \int_{0}^{1}\left(\alpha\left|\frac{d \mathbf{r}(s)}{d s}\right|^{2}+\beta\left|\frac{d^{2} \mathbf{r}(s)}{d s^{2}}\right|^{2}\right) d s
$$

where $\alpha$ and $\beta$ are weighting parameters. The first term, also known as the tension energy, prevents the contour from sticking to isolated points by penalizing stretching of the contour. The second term, known as the bending energy, measures the smoothness, e.g. by penalizing sharp corners. More complex energy terms, for example shape regularization based on Fourier descriptors, have also been reported in literature. ${ }^{10-12}$ 
The external energy is derived from the image, such that the contour will be attracted to features of interest. Given a gray level image $I(x, y)$, a common external energy is defined as:

$$
E_{\text {ext }}[\mathbf{r}(.)]=-\int_{0}^{1} F(\mathbf{r}(s)) d s
$$

where $F(x, y)$ is a feature map. Common features of interest are edges, e.g.

$$
F(x, y)=|\nabla I(x, y)|^{2} \text { or } F(x, y)=\left|\nabla\left(G_{\sigma}(x, y) * I(x, y)\right)\right|^{2}
$$

where $\nabla$ is the gradient operator, $G_{\sigma}(x, y)$ a 2D Gaussian kernel with standard deviation $\sigma$ and where $*$ is the convolution operator.

Eq. (2) can be minimized by treating $\mathbf{r}(s)$ as a function of time, i.e. $\mathbf{r}(s, t)$. This requires finding $\mathbf{x}(s, t)$ and $\mathbf{y}(s, t)$ such that

$$
\begin{aligned}
\frac{\partial x(s, t)}{\partial t} & =\alpha \frac{\partial^{2} x(s, t)}{\partial s^{2}}-\beta \frac{\partial^{4} x(s, t)}{\partial s^{4}}+\frac{\partial F(\mathbf{r}(s, t))}{\partial x} \\
\frac{\partial y(s, t)}{\partial t} & =\alpha \frac{\partial^{2} y(s, t)}{\partial s^{2}}-\beta \frac{\partial^{4} y(s, t)}{\partial s^{4}}+\frac{\partial F(\mathbf{r}(s, t))}{\partial y}
\end{aligned}
$$

vanish for all $s$. This can be achieved by iteratively solving a discretization of $s$ using a finite difference approach. ${ }^{2,13}$

The external energy term defined in the previous section usually requires a good initialization, close to the object boundary, in order to achieve correct convergence. This limitation is caused by the nature of the external energy term, which is typically non-flat only in the proximity of the object's boundary. To overcome this problem, Xu and Prince ${ }^{14}$ proposed the use of an external force field, $\mathbf{v}(x, y)=(P(x, y), Q(x, y))$, where $P(\mathbf{r}(s, t))$ and $Q(\mathbf{r}(s, t))$ replace the partial derivatives of $F(\mathbf{r}(s, t))$ in Eq. (6),i.e.

$$
\frac{\partial \mathbf{r}(s, t)}{\partial t}=\alpha \frac{\partial^{2} \mathbf{r}(s, t)}{\partial s^{2}}-\beta \frac{\partial^{4} \mathbf{r}(s, t)}{\partial s^{4}}+\mathbf{v}(\mathbf{r}(s, t))
$$

The vector field $\mathbf{v}(.,$.$) is calculated by minimizing the following energy functional:$

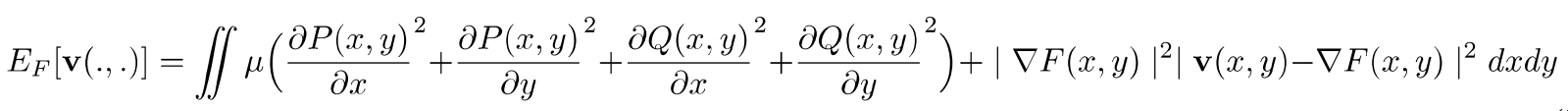

where $\mu$ is a non-negative parameter expressing the degree of smoothness imposed on the field $\mathbf{v}$ and where $\mathrm{F}$ is a feature map such as in Eq. (5). The first term of Eq. (8) keeps the field $\mathbf{v}$ smooth, whereas the second term forces the field $\mathbf{v}$ to resemble the original edge force in the neighborhood of edges. This external force is called Gradient Vector Flow (GVF) field. The force field with minimal energy can be found using gradient descent optimization. ${ }^{14}$

\subsection{Leaf Detection}

In previous work ${ }^{15}$ the authors showed that leaf segmentation in time lapse sequences using active contours is prone to errors, due to the low temporal resolution. Instead they proposed to work in two steps: the first step moves the snake without deforming it, while only in the second step the actual deformation is done. This first step results in a rough detection of the leaves, which can be further deformed for the actual segmentation. This step can be formulated in the active contour framework by defining a new internal energy:

$$
E_{\text {int }}[\mathbf{r}(.)]=\int_{0}^{1} \gamma\left\|T(s ; \mathbf{r}(.))-T\left(s ; \mathbf{r}_{\mathbf{0}}(.)\right)\right\| d s
$$

Where $\mathbf{r}_{\mathbf{0}}($.$) represents the initialization of the active contour and \gamma$ is a weighting parameter. The function $T(. ;$.$) trans-$ poses a curve such that the center of mass of the curve equals zero, i.e.

$$
T(s ; \mathbf{x}(.))=\mathbf{x}(s)-\int_{0}^{1} \mathbf{x}(s) d s
$$




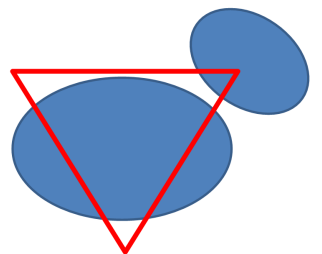

(a)

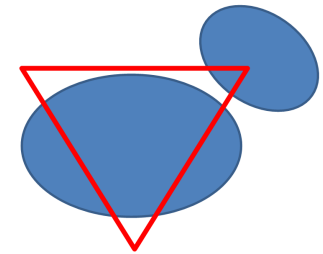

(b)

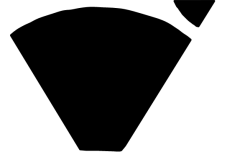

(c)

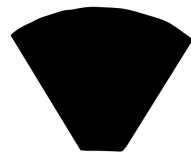

(d)

Figure 3. The four steps of leaf detection: (a) initialization, (b) rigid active contour optimization, (c) fusion with the rosette segmentation and (d) discarding small false segments.

If the weight of the internal energy, $\gamma$ in eq. (9) is high, i.e. $\gamma \gg$ average external force working on the active contour, then this optimization results in translating the active contour as a rigid body. The direction of the translation is given by the average external force working on the active contour, i.e.

$$
\frac{\partial \mathbf{r}(s, t)}{\partial t}=\int_{0}^{1} \mathbf{v}(\mathbf{r}(s, t)) d s
$$

where $\mathbf{v}$ is the gradient vector flow external force as described in eq. (8). Once this rigid snake stabilizes, the pixels enclosed by the snake can be considered to be a rough detection of the leaf. This detection can be further refined by combining it with the segmentation result of the rosette (see section 3). Since a leaf is part of the rosette, each pixel enclosed by the active contour but not belonging to the rosette segment can be discarded. This may result in a group of detected pixels which are no longer connected. However the snake algorithm needs a single curve which can be deformed, therefore we will take the boundary of the largest connected component. In Fig. 4.2 the consecutive steps are shown for a synthetic example. In Fig. 4.2 (a) the blue ellipses represent the leaves, whereas the red triangle corresponds to the initialization of the active contour, e.g. the resulting contour from a previous frame. In Fig. 4.2 (b) the initial contour has been translated according to the optimization given by eq. (11). Assume that the leaves are correctly segmented by the rosette segmentation, then the pixels both belonging to the rosette segment and enclosed by the active contour are shown in black in Fig. 4.2 (c). In Fig. 4.2 (d) only the largest connected component has been kept as the detection of the leaf. The boundary of this connected component will be used as an initialization for the actual leaf segmentation as described in the next section.

\subsection{Leaf Segmentation}

The leaf detection step results in a proper initialization for the segmentation of each individual leaf. However, low contrast and difference in leaf intensity hampers the use of active contour segmentation. An example of this problem and the effect on the segmentation result are shown in Fig. 4.3. Fig. (a) shows two objects with different intensity, e.g. black and light gray. The red ellipse is the initialization of the active contour for the gray object. The boundary of the black object will have a stronger edge response than the gray object, which affects the GVF force (see subsection 4.1). This influence is shown in Fig. 4.3 (b) where it is clear that the black object has a much wider capturing range than the gray object. This results in erroneous segmentation results, since a part of the active contour is attracted to the wrong object. This can be seen in Fig. 4.3 (c).

The gradient vector flow force tries to extend the capturing range of the active contour by assuming that the force field should be smooth except in regions where there are clear edges. This smoothness is enforced over the complete image, i.e. the capturing range is extended over the full image, whereas we know in advance that all initial contours lie within the detected rosette segments. Instead we propose to use a force field which is smooth over a predefined shape. This is calculated by minimizing the following energy function:

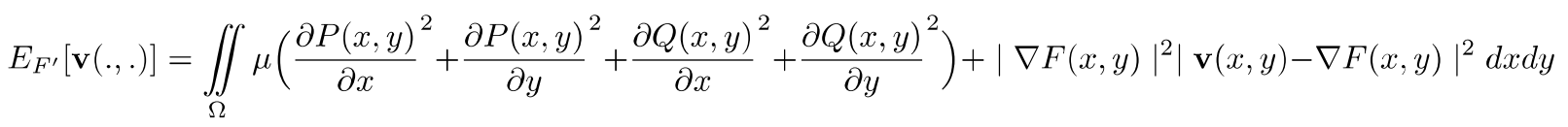



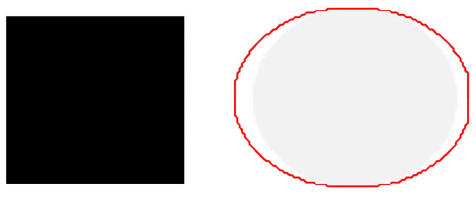

(a)

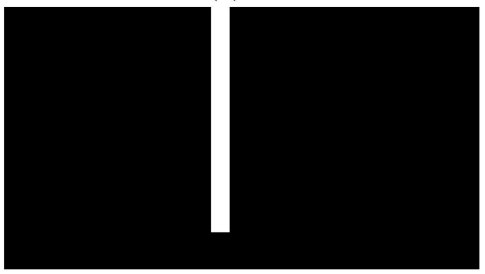

(d)

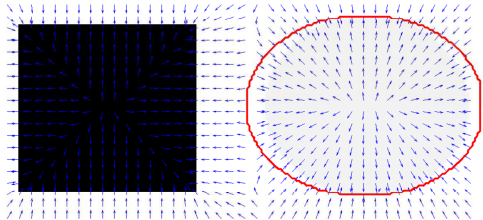

(b)

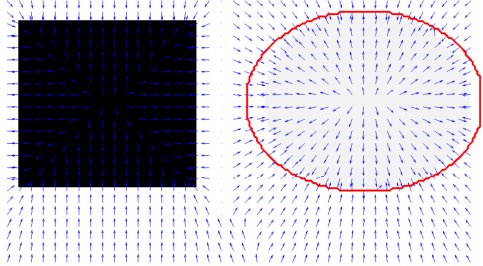

(e)

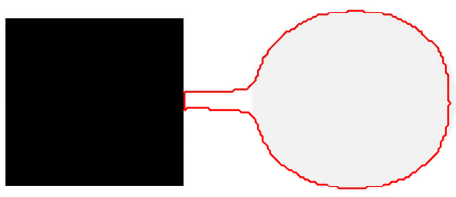

(c)

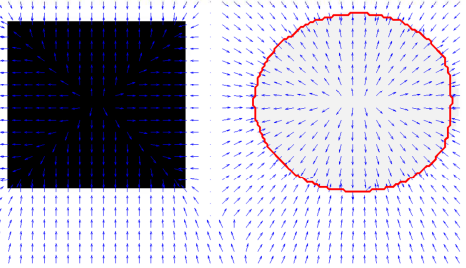

(f)

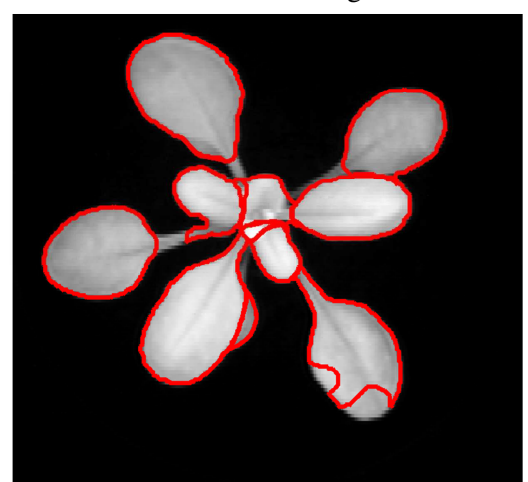

(a)

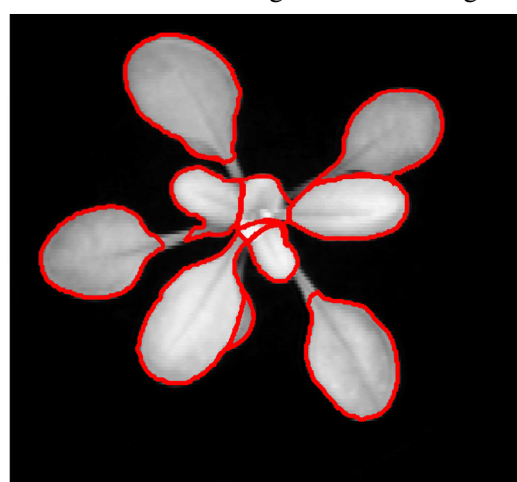

(b)

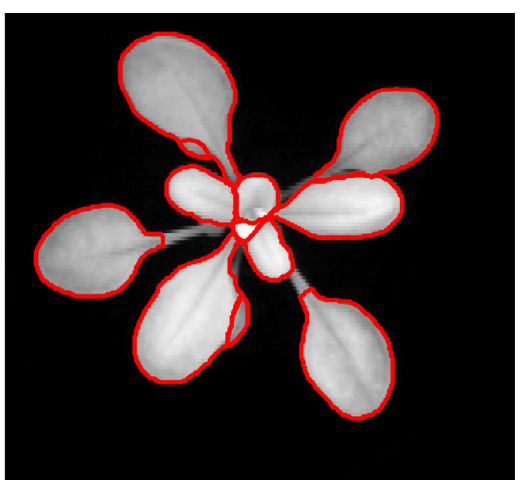

(c)

Figure 5. An example of segmentation using (a) GVF, (b) moving and (c) the proposed SCGVF snakes.

where $\Omega$ are the points belonging to the rosette segments. At points which do not belong to the rosette segment, i.e. the background, the force is set equal to the zero vector. Since this force field is calculated based on the GVF energy function over the shape of the rosette, we will refer to this energy function as shape constrained gradient vector flow (SCGVF) In Fig. 4.3 (f) the influence of this force field is shown for the same segmentation problem as in Fig. 4.3 (a). Fig. 4.3 (d) shows the mask representing the rosette segments in black. Note that this is a very rough approximation of the segments, but nevertheless it will be sufficient to achieve correct segmentation. In Fig.4.3 (e) the SCGVF force field can be seen. The capturing range of the black object is clearly less than in Fig. 4.3 (b), since the force has to work around the boundary of the rosettes shape. In (f) the segmentation result of the active contour using the SCGVF is shown.

\section{RESULTS}

Fig. 5 shows an example of active contour tracking of multiple leaves. The images show the 18th frame. The first processed frame, i.e. the last time frame of the sequence, was initialized with the same manual delineation of the leaves. Fig. 5 (a) shows the result of the regular GVF snakes. This method results in bad segmentation as can be seen in the example. This is mainly due to the low temporal resolution, the leaf moves too far for the active contour to optimize correctly. In Fig. 5 (b) the result of the so called moving snake is shown. ${ }^{15}$ These snakes where developed for tracking leaves in time lapse sequences. Therefore the method has less problems with the low temporal resolution. Nevertheless does the method still result in erroneous results, e.g. a small partially occluded segment is no longer found or parts of the stalk are merged with a leaf. The proposed SCGVF snake result is shown in Fig. 5 (c). As can be seen does this result in proper segmentation of the frame.

In order to quantitatively validate the proposed method, a time lapse sequence of 20 frames was annotated. The annotation of each frame was the result of watershed segmentation ${ }^{16}$ manually corrected by a human operator. The watershed 


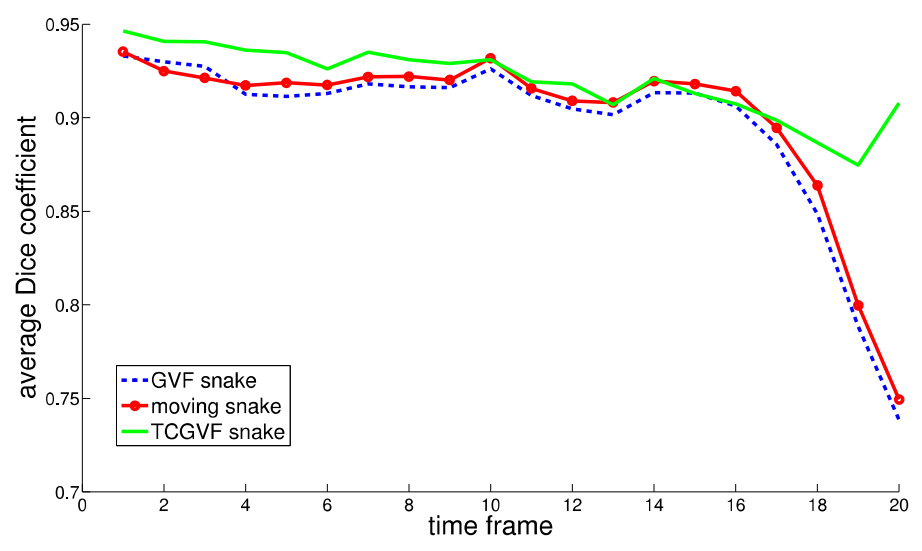

Figure 6. The average Dice coefficient for each frame.

algorithm missed on average 3 leaves, found 1 false segment and had 6 over or under segmentation errors for each frame. This makes it clear that the watershed algorithm might be useful as a start for a human operator, but is no option for real automatic analysis of leaf fluorescence time lapse sequences.

For the validation of the segmentation, the Dice coefficient is used. If $S$ is the resulting segment from the active contour, i.e. the region enclosed by $\mathbf{r}(s)$, and GT the ground truth segment, then the Dice coefficient between S and GT is defined as:

$$
d(S, G T)=\frac{2 \operatorname{Area}(S \wedge G T)}{\text { Area }(\mathrm{S})+\operatorname{Area}(G T)}
$$

where $S \wedge G T$ consist of all pixels which both belong to the detected segment as well as to the ground truth segment. If $S$ and GT are equal, the Dice coefficient is equal to one. The Dice coefficient will approach zero if the regions hardly overlap.

In Fig. 6 the average dice coefficient is plotted for each time frame. The first frame was initialized by the boundaries of the ground truth segments. As can be seen does the proposed method result in good tracking results, whereas the regular GVF or moving snake clearly are hampered by error propagation. The error propagation of the state of the art methods can be observed by the fast decreasing Dice coefficient starting around frame 16.

\section{CONCLUSION}

This paper proposes an automated method to measure individual leaf fluorescence in time lapse sequences. The proposed method consists of three steps: rosette segmentation, leaf detection and leaf segmentation. Both the leaf detection as the leaf segmentation are an extension of GVF snakes. Experiments showed that the proposed shape constrained GVF snakes are more robust for low temporal resolution sequences than GVF snakes. Although this paper focuses on leaf tracking, the method has generic value for applications where a rough segmentation result is available. This rough segmentation can then be incorporated in the SCGVF in order to improve the snake optimization.

\section{ACKNOWLEDGMENTS}

This research has been made possible by the Institute for the Promotion of Innovation by Science and Technology in Flanders (IWT). The authors wish to thank Laury Chaerle for setup and image acquisition of the time lapse sequence.

\section{REFERENCES}

1. L. Chaerle and D. Van Der Straeten, "Imaging techniques and the early detection of plant stress," Trends Plant Sci 5(11), pp. 495501, 2000.

2. M. Kass, A. Witkin, and D. Terzopoulos, “Snakes: active contour models," International journal of computer vision, pp. 321-331, 1988. 
3. G. Tsechpenakis, K. Rapantzikos, N. Tsapatsoulis, and S. Kollias, "A snake model for object tracking in natural sequences," Signal Processing-Image Communication 19(3), pp. 219-238, 2004.

4. M. A. Charmi, S. Derrode, and F. Ghorbel, "Fourier-based geometric shape prior for snakes," Pattern Recognition Letters 29(7), pp. 897-904, 2008.

5. T. Chan and L. Vese, "An active contour model without edges," Scale-Space Theories in Computer Vision 1682, pp. 141-151, 1999.

6. R. Goldenberg, R. Kimmel, E. Rivlin, and M. Rudzsky, "Fast geodesic active contours," IEEE Transactions on Image Processing 10(10), pp. 1467-1475, 2001.

7. T. F. Chan, S. Esedoglu, and M. Nikolova, "Algorithms for finding global minimizers of image segmentation and denoising models," Siam Journal on Applied Mathematics 66(5), pp. 1632-1648, 2006.

8. X. Bresson, S. Esedoglu, P. Vandergheynst, J. P. Thiran, and S. Osher, "Fast global minimization of the active contour/snake model," Journal of Mathematical Imaging and Vision 28(2), pp. 151-167, 2007.

9. N. Otsu, "Threshold selection method from gray-level histograms," IEEE Transactions on Systems Man and Cybernetics 9(1), pp. 62-66, 1979 .

10. M.-A. Charmi, S. Derrode, and S. Ghorbel, "Fourier-based geometric shape prior for snakes," Pattern Recognition Letters 29, pp. 897-904, 2008.

11. A. Goobic, M. Welser, S. Acton, and K. Ley, "Biomedical application of target tracking in clutter," Proc. 35th Asilomar Conference on Signals, Systems and Computers 1, pp. 88-92, 2001.

12. M. Rochery, I. H. Jermyn, and J. Zerubia, "Higher order active contours," Int. J. Comput. Vision 69(1), pp. 27-42, 2006.

13. L. Cohen, "On active contour models and balloons," CVGIP: Image Understanding 53, pp. 211-218, 1991.

14. C. Xu and J. Prince, "Snakes, shapes and gradient vector flow," IEEE transactions on image processing 7, pp. 359-369, 1998. http://iacl.ece.jhu.edu/projects/gvf/.

15. J. De Vylder, D. Ochoa, W. Philips, L. Chaerle, and D. Van Der Straeten, "Tracking multiple objects using moving snakes," in 16th International Conference on Digital Signal Processing (DSP2009), (Santorini, Greece), jul. 2009.

16. R. Gonzales, R. Woods, and S. Eddings, Digital image processing using Matlab, Pearson/Merrill/Prentice Hall, 2004. 